\title{
Measuring Legislative Boundaries
}

\author{
Christopher P. Chambers* and Alan D. Miller ${ }^{\dagger \ddagger}$ \\ May 11, 2013
}

\begin{abstract}
We introduce the first family of district compactness measures that can incorporate a wide range of internal geographic features. The measures in this family are the probability that a district contains an admissible path between a randomly selected pair of people. The measure can account for roads, travel time, political boundaries, and prior districts. This family of measures includes the path-based measure of Chambers and Miller (2010).
\end{abstract}

\section{Introduction}

Two hundred years ago, legislators discovered that the ultimate composition of a legislature is not independent of the means through which district boundaries are drawn. Hoping to stave off unemployment, legislators learned to master the art of gerrymandering: carefully drawing district boundaries to increase their electoral chances and political power. Although quickly recognized as a danger, the gerrymander has been hard to kill. It has survived to this day as

*Department of Economics, University of California, San Diego, 9500 Gilman Drive, \#0508, La Jolla, CA 92093-0508. Email: cpchambers@uscd.edu Web: http://econ.ucsd.edu/ cpchambers/

${ }^{\dagger}$ Faculty of Law and Department of Economics, University of Haifa, Mount Carmel, Haifa, 31905, Israel. Email: admiller@econ.haifa.ac.il Web: http://econ.haifa.ac.il/ admiller/

${ }_{\ddagger}^{\ddagger}$ The authors would like to thank Tiberiu Dragu, Paul Edelman, Bernhard Ganglmair, Daniel Goroff, Matt Spitzer, Avraham Tabbach, Daniel Ullman, as well as an anonymous referee, the Russell Sage Foundation, and participants at the 2009 Joint Mathematics Meetings. 
an endless source of study for academics and as a constant target of political reformers.

Scholars and practitioners have proposed a number of methods to measure gerrymandering. Among the most prominent of these are measures of compactness, which assign a real number to each geographic district, or districting plan; the idea being that a lower number corresponds to a more heavily gerrymandered district. Existing measures take into account the shape and, occasionally, the population of the districts. As a simplification, all ignore geographic features internal to the districts, such as mountain ranges and county boundaries.

However, there is a gap between the measures of compactness, on one hand, and the redistricting reality, on the other. These internal geographic features are not ignored by those responsible for drawing the actual district lines. Such features are often invoked as a justification for oddly shaped districts, whether for legitimate reasons or as an excuse. As a practical matter, the laws of forty-four states require districting plans to respect pre-existing political subdivisions, such as counties, municipalities, and voting precincts.

To bridge this gap, we introduce a new class of path-based compactness measures. As a special case, this class includes the path-based measure developed by Chambers and Miller (2010). To our knowledge, these are the first compactness measures that can incorporate a wide range of internal geographic features. While our primary focus is on the geographic aspects of redistricting, we note that our approach may also be used to analyze other justifications for oddly shaped districts, including the requirement in some states to preserve the cores of prior districts.

\subsection{Compactness Measures}

In the past fifty years, a wide array of methods have been proposed to measure district compactness. Roughly speaking, these methods can be separated into three classes, dispersion, perimeter, and bizarreness measures.

Dispersion measures assess the extent to which a district is spread out over a large area. These include (i) comparing the length of the district to its width (Harris, 1964) ${ }^{1}$ (ii) comparing the area of the district to the area of the smallest circle containing the district (Reock, 1961), and (iii) making comparisons based off of the district's center of gravity (Boyce and Clark, 1964;

\footnotetext{
${ }^{1}$ See also Iowa Code $\S 42.4(2009)$.
} 
Kaiser, 1966). These last two approaches can be adapted to account for the distribution of the district's population (Hofeller and Grofman, 1990; Weaver and Hess, 1963).

There have also been attempts to measure entire districting plans instead of individual districts. In particular, Papayanopoulos (1973) proposed summing the distances between all pairs of individuals contained within the same district. $^{2}$ These districting plan measures have intuitive appeal in that redistricters must choose between districting plans and do not choose individual districts in isolation. In this context, Fryer and Holden (2011) argue that it may be misleading to study the choice of a single district without considering other districts affected by the plan.

However, all districting-plan measures suffer from the scale-invariance problems identified by Young (1988). Districting-plan measures may also be of limited value in legal cases where the appropriate unit for analysis is the individual district, as is the case with claims arising under Section 2 of the Voting Rights Act. In cases in which the courts would analyze an entire districting plan (e.g. Georgia v. Ashcroft, 539 U.S. 461 (2003), involving a retrogression claim brought under Section 5 of the Voting Rights Act), we advocate using, as the measure of the districting plan, the score of the least-compact district in the state. This approach measures the districting plan as a function of the individual district scores and combines the advantages of a districting-plan measure with those of a scale-invariant district-specific measure. ${ }^{3}$ For more on districting-plan measures, see Chambers and Miller (2010).

Perimeter measures use the length of district boundaries as a proxy for compactness. The most common such measure compares the length of the district perimeter to the square-root of its area (Schwartzberg, 1966; Cox, 1927; Weaver and Hess, 1963; Polsby and Popper, 1991). The state of Colorado measures entire districting-plans by summing the length of all district boundaries. ${ }^{4}$

\footnotetext{
${ }^{2}$ For a more recent proposal that sums the squares of the distances, see Fryer and Holden (2011).

${ }^{3}$ The districting-plan measures proposed by Papayanopoulos (1973) and Fryer and Holden (2011) work in a similar away, in that they are formed by calculating a score for each district (e.g. the sum of all pairwise distances between individuals in the district), and then these scores are summed over all districts. In the case of Fryer and Holden (2011), the resulting number is then multiplied by a constant - the inverse of the score of the most compact feasible districting plan - so that the optimal plan has a measure of one).

${ }^{4}$ See Colo. Const. Art. V., Section 47.
} 
Bizarreness measures use the mathematical concept of convexity to describe nice districts. A district is convex if a rubber band will fit snugly around its border. Squares, triangles, and circles are convex; hourglasses, crescent moons, and stars are not. Taylor (1973) measures convexity by comparing the number of inward-bending angles on the district's perimeter to the number of outward-bending angles. (A convex shape has no inward bending angles.) Chambers and Miller (2010) look at whether the shortest path connecting two individuals remains within the district. (The shortest path will always remain inside a convex district.)

Serious flaws have been found in most of these approaches. We will not describe all of the problems here. Instead, we will focus on one serious limitation of all existing methods. For an in-depth review of existing compactness measures, see surveys by Young (1988), Niemi, Grofman, Carlucci, and Hofeller (1990), Altman (1998), and Chambers and Miller (2010).

\subsection{Internal Geographic Constraints}

A major limitation of existing district compactness measures is that none are able to account for the presence of geographic features such as mountain ranges and county lines. Until recently, no district measures were able to incorporate state boundaries. The first such measure was introduced by Chambers and Miller (2010). This measure is the first under which a district is not penalized because it was drawn to satisfy the obvious constitutional requirement that it remain within the state. ${ }^{5}$

We introduce a new family of measures which can account for exogenous internal geographic features, both physical (e.g. mountains, lakes, rivers, and roads) and political (e.g. county, municipal, and precinct boundaries). This family of measures can also incorporate some political concerns relevant to gerrymandering, such as the requirement in some states to preserve the cores of prior districts. This new family of measures includes the original path-based

\footnotetext{
${ }^{5}$ The ability to incorporate state-boundaries in a compactness measure is necessary if one wishes to make comparisons either (i) between districts in different states or (ii) between districts in different areas of the same state. Without such a measure, one cannot use a precedent regarding a district in one state (e.g. North Carolina) to determine the legality of a contested district in a different state (e.g. Texas). Similarly, two potential districts in the same state which are not similarly affected by the state's boundaries would otherwise be incomparable. In this case, a districting-plan measure based off of the individual district scores would not be meaningful.
} 
measure of Chambers and Miller (2010) as a special case.

The original path-based measure of Chambers and Miller (2010) is simple to explain. Because there are several variants, we shall focus on the population weighted measure which takes exogenous state boundaries into account. To compute the measure, pick any pair of residents in a district, and find the shortest path between them, distance-wise, which remains in the state. The path-based measure is the probability that, if we select residents uniformly and at random, the shortest path between them is contained entirely within the district. An example is shown in Figure 1: Nevada's Second Congressional District contains the shortest paths in the state between the towns of Paradise and Bunkerville, and between Bunkerville and Laughlin, but not between Paradise and Laughlin.

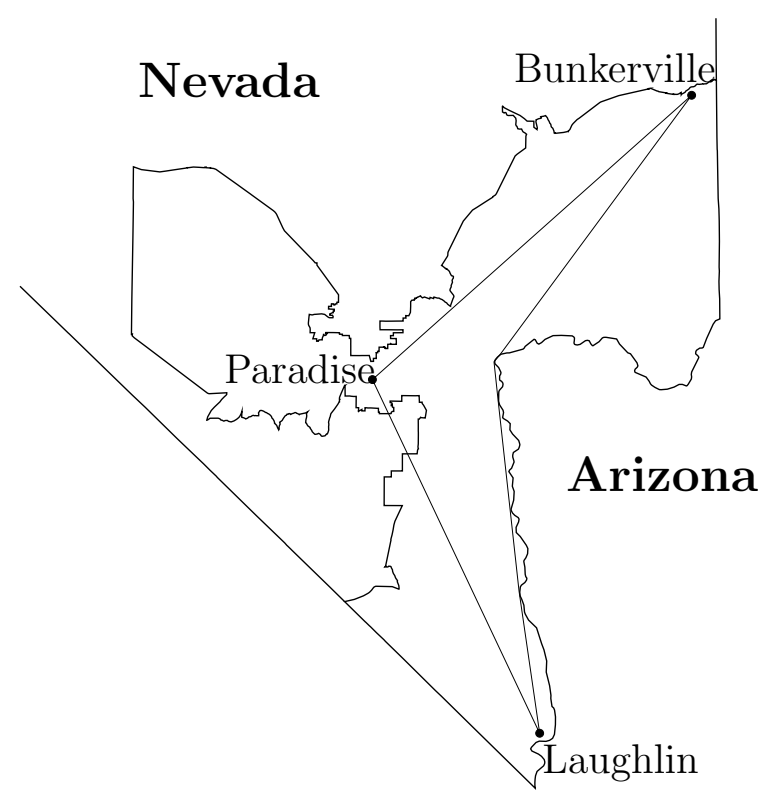

Figure 1: Nevada 2nd Congressional District, 109th Congress

The shortest path computed by Chambers and Miller (2010) was distancebased. Of course, two people on opposite sides of the Grand Canyon may be close in terms of distance, but very far in terms of difficulty of travel. To account for this problem, we aim to generalize this notion of the shortest path. The shortest path between two people on opposite sides of the Grand Canyon may be a path that goes around it. 
To this end, we introduce a notion of an admissible path. An admissible path is a path between two points that is objectively determined, according to some method, to be a path of minimal cost. ${ }^{6}$ Examples of classes of admissible paths include, but are not limited to, (i) minimal distance paths following roads and (ii) minimal paths induced by some metric — such as travel time. Classes can also be defined to take into account pre-existing political subdivisions (such as county, municipal, and precinct boundaries). Our measure, then, is the probability that a district contains an admissible path between a randomly selected pair of people. This measure assigns to each district a score between zero and one, with scores closer to one representing more compact districts.

\subsection{Districting Principles}

Individuals charged with the task of redrawing legislative district lines are the servants of many masters. State laws impose various restrictions, often vague and at times contradictory, such as that districts be compact and contiguous, or respect county boundaries, or preserve communities of interest. The Voting Rights Act of 1965 requires that districting plans may not have the purpose or effect of denying or abridging the right to vote on account of race or color."7 In addition, the Equal Protection Clause of the Fourteenth Amendment to the U.S. Constitution has been held to require strict scrutiny when redistricting legislation "is so extremely irregular on its face that it rationally can be viewed only as an effort to segregate the races for purposes of voting, without regard for traditional districting principles and without sufficiently compelling justification." "We will explain several districting principles, including compactness, preservation of political subdivisions, and preservation of cores of prior districts, and will analyze the extent to which our proposed family of measures can account for these principles.

We emphasize that our argument is theoretical, and not empirical. Our aim is to show that we have a theoretically sound tool for measuring compliance with these legally mandated principles. While several examples from existing districts are provided for illustration (Figures 1-4), ${ }^{9}$ such examples are of limited value in evaluating the measure. Districts would presumably change

\footnotetext{
${ }^{6}$ There may be more than one admissible path.

${ }^{7}$ Voting Rights Act of 1965, Public Law 89-110, 42 U.S.C. $§ 1973-1973 a a-6$.

${ }^{8}$ Shaw v. Reno, 509 U.S. 630, at 642 (1993).

${ }^{9}$ The districts in these examples were chosen because they provide clear illustrations of the specific features of the measure that we wish to discuss.
} 
if a different measure (or set of measures) was used. We expect that use of the measures described in this paper would affect the district compactness scores substantially in some states and much less so in others. In these latter states, low scores might indicate that otherwise oddly-shaped districts cannot be defended using this approach.

First and foremost among these principles is legislative district compactness. There are many different concepts of compactness, centered around ideas of dispersion, long perimeter, and bizarreness. Our family of measures can clearly be used to measure compactness - as noted above, it includes the path-based measure introduced by Chambers and Miller (2010), which identifies bizarrely shaped districts.

This compactness measure can be modified to incorporate several types of geographical constraints using the notion of admissible paths. We will describe two such possibilities here. First, we can define the set of paths to be the existing network of roads, and declare a path between two people to be admissible if it is the shortest road between them. Second, we can choose the shortest path using travel time as opposed to physical length. In both cases, readily available mapping software can be used to determine the shortest paths. In principle, other existing measures can also be redefined in this way, including those proposed by Papayanopoulos (1973) and Fryer and Holden (2011).

The road-based or travel time-based measures may lead to a new interpretation of "freeway districts", such as the proposed North Carolina Twelfth Congressional District struck down by the Supreme Court in Shaw v. Reno. ${ }^{10}$ Approximately 160 miles long, the district followed much of U.S. Interstate 85 for much of its length, picking up communities of voters adjacent to the freeway along the way. A freeway district may, in fact, contain the shortest paths (in terms of time-travel) between many of its residents. ${ }^{11}$

Alabama's Sixth and Seventh Congressional Districts are shown in Figure 2. The Seventh district connects Birmingham, Alabama's largest city, to points further south and west by means of a narrow corridor that contains U.S. Interstate 20. The shortest paths in terms of travel time are shown be-

\footnotetext{
${ }^{10}$ Shaw v. Reno, 509 U.S. 630 (1993).

${ }^{11}$ The district invalidated in Shaw $v$. Reno would be unlikely to receive a high score; as it did not follow the freeway for all its length, and it did contain all of the freeway. As the court noted "Northbound and southbound drivers on I-85 sometimes find themselves in separate districts in one county, only to 'trade' districts when they enter the next county." 509 U.S. 630 , at 636.
} 
tween Birmingham and a number of cities and towns in other parts of the district. ${ }^{12}$ All of these paths remain in the district, even though the straight lines connecting Birmingham to these places would cross into other districts. As a result, the Seventh district would likely be considered more compact were the travel time metric to be used.

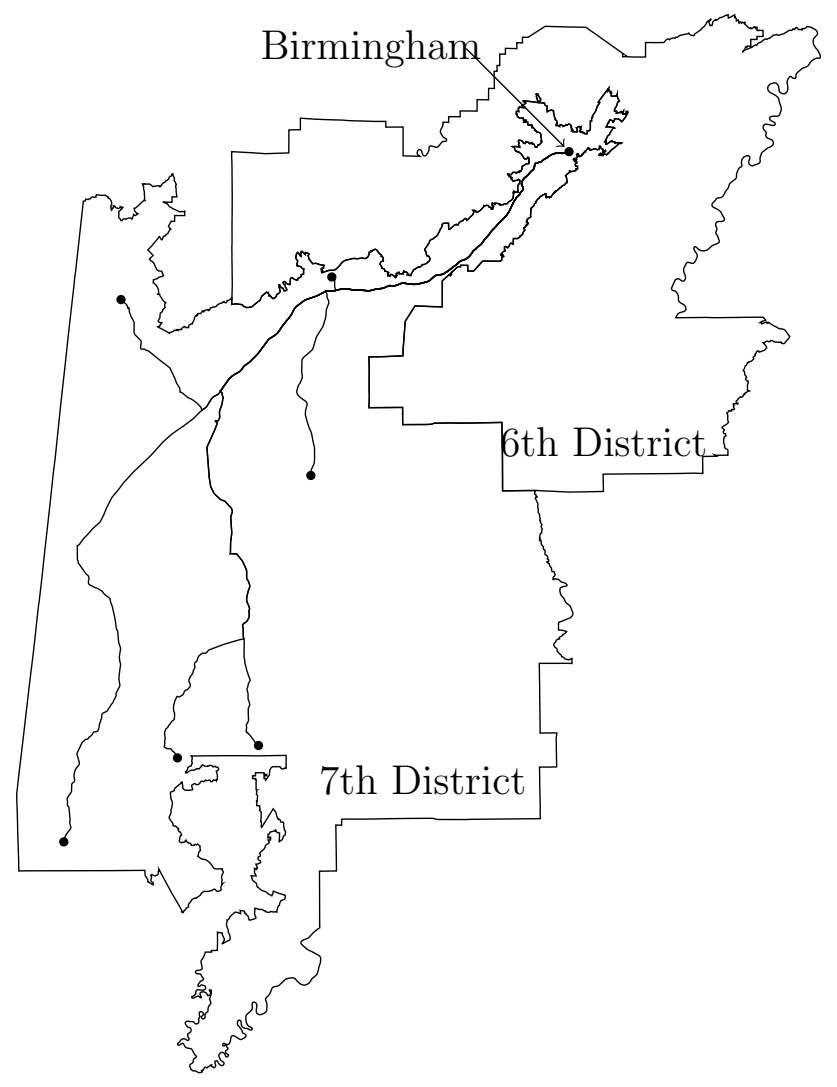

Figure 2: Alabama 6th and 7th Congressional Districts, 109th Congress

The inclusion of Birmingham through this narrow corridor comes at a cost. Often, the fastest way to travel between points in the Sixth district is through the freeways in this corridor. As a result, the Sixth district would be considered relatively non-compact. There are two lessons. First, when evaluating a districting plan, it is important to consider the scores of all districts. Second, if a freeway is used to connect people in distant parts of the state, then the

\footnotetext{
${ }^{12}$ The shortest paths in terms of travel time were calculated using Google Earth, available at http://www.google.com/
} 
score of another district may suffer. Residents of the other district may also use the freeway as part of their shortest path.

The road-based or travel time-based measures may also lead to a new interpretation of "bridge and tunnel" districts such as Maryland's Second and Third Congressional Districts, connected respectively by the Fort McHenry Tunnel and the Francis Scott Key Bridge. It may be faster to go over or under a bay than to travel around it. ${ }^{13}$

A second principle is that legislative districts should, to the extent possible, preserve pre-existing political subdivisions. The laws of forty-four of the fifty states contain provisions requiring redistricters to preserve county, municipal, and precinct boundaries when redrawing the district lines. (NCSL, 2009) The simplest solution is to declare a path admissible if it is the shortest path remaining entirely within the county, city, or voting precinct. This leads to the possibility, of course, that there may be multiple admissible paths - one could define both the shortest path in the state and the shortest in the county to be admissible paths, depending on the objectives. Figure 3 shows Arkansas' Third Congressional District. The irregular borders on the north east and southern portions of the district (shown in bold) follow the county boundaries.

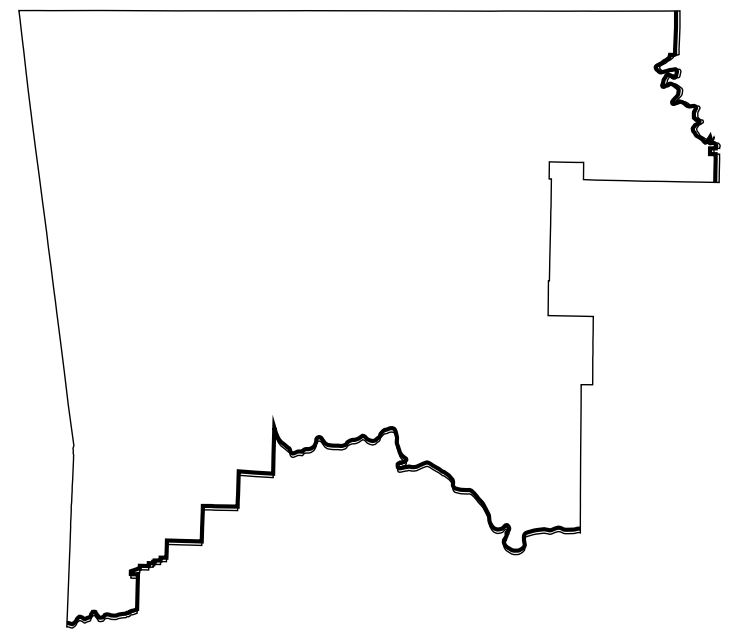

Figure 3: Arkansas 3rd Congressional District, 109th Congress

\footnotetext{
${ }^{13}$ We note, however, while it may be possible to construct compact "bridge and tunnel" districts under a road-based metric, neither of these Maryland districts would be considered compact by such a measure. Both districts are extremely bizarre even if the roads are taken into account.
} 
A third principle is that districting plans should preserve the cores of prior districts. The new district should be as similar as possible to the former district. This objective can be achieved by declaring a path between two points to be admissible if it was the shortest path in the previous district or, if the two points were not in the same district, if it is the shortest path in the state. The method would allow districts to be similar to the previous districts (regardless of their intrinsic shape) while limiting the ability of the redistricters to gerrymander.

For example, consider Alabama's First Congressional District, shown in Figure 4. After the 2000 Census, the First district decreased in terms of area because of a relative decline in population. The revised district is almost entirely contained within the original district, but has a more bizarre shape. One can see that the odd shape of the revision can not readily be justified by the claim that the district was drawn to preserve the core of the prior district. The newer district does not contain the shortest path between Fulton and Buena Vista in the older district.

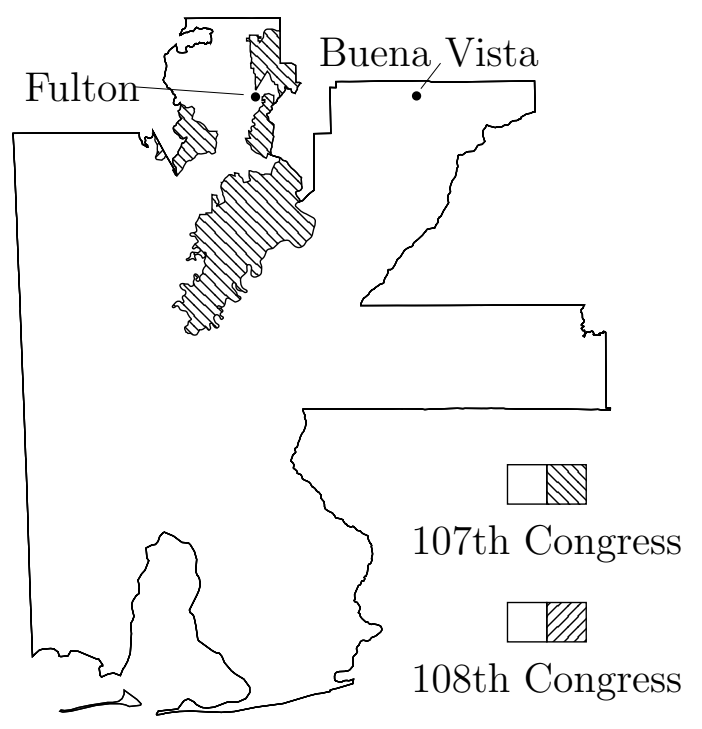

Figure 4: Alabama 1st Congressional District, before and after redistricting

Other principles include compliance with the Voting Rights Act of 1965, preserving "communities of interest", and the contradictory goals of protecting incumbents and increasing the competitiveness of elections. These objectives are strictly political in nature and can not be accounted for using a compact- 
ness measure. ${ }^{14}$

\section{The Admissible Path Measure}

A state $Z$ is a compact subset of $\mathbb{R}^{2}$ which has a path-connected interior and is the closure of its interior. ${ }^{15} \mathrm{~A}$ district $K$ is simply a closed subset of $Z$ which has a path-connected interior, and which is the closure of its interior. The set of all possible districts in $Z$ will be denoted $\mathcal{K}_{Z}$. For $x, y \in Z$, let $\mathcal{P}_{Z}(x, y)$

\footnotetext{
${ }^{14}$ In general, the question of whether a districting plan violates the Voting Rights Act of 1965 (whether it has the effect of denying members of a protected minority group the right to participate in the political process) is judged with other tools. For example, the number of majority-minority seats is often used as a proxy for minority voting power. However, while a compactness measure will not tell us whether a voting plan complies with the act, we may be able to evaluate seemingly odd-shaped districts to check whether compliance with the Act is merely an excuse to gerrymander.

Implicit within the Voting Rights Act is the idea that there is a group of minority voters with similar interests who should be kept together in a single district. Our model could be generalized so that one could define all paths between members of this group to be admissible; the resulting measure describes how gerrymandered the district is with respect to non-members of the group. One could use a similar approach to measure gerrymandering with respect to members of the group. If the former is substantially more gerrymandered than the latter, this may indicate a significant amount of gerrymandering not directly caused by the desire to keep members of this minority group together. A high score, of course, will not necessarily protect the district from claims under the Equal Protection Clause of the Fourteenth Amendment. We do not address the question of whether such an approach is plausible - it seems unlikely that a court would use only non-minorities to see whether a district is gerrymandered - but we simply note that there are a variety of measures which can be constructed depending on the specific interpretation of the Voting Rights Act.

While using this approach, it is important to pick the minority group appropriately. Following recent the Supreme Court decisions in League of United Latin American Citizens v. Perry, 548 U.S. 399 (2006), it might not be appropriate to define all paths between members of a minority group to be admissible, at least in a state as large as Texas. On the other hand, it might be appropriate to define a path as admissible if it is between two members of the group and is less than forty miles in length.

The principle that plans preserve "communities of interest" is similar. This vague concept means that it is legitimate to draw legislative district lines to protect certain political interests. In principle these may be interests of any group, not necessarily a protected racial or ethnic minority. For example, one might define all paths between orthodox Jews in Williamsburg, New York, to be admissible. See United Jewish Organizations of Williamsburgh, Inc. v. Carey, 430 U.S. 144 (1977).

${ }^{15}$ Compactness is with respect to the Euclidean topology and does not refer to political compactness.
} 
denote the set of continuous paths ${ }^{16} g:[0,1] \rightarrow Z$ for which $g(0)=x$ and $g(1)=y$. For all $x, y \in Z$ distinct, associate a set $\mathcal{A}_{Z}(x, y) \subset \mathcal{P}_{Z}(x, y)$ of admissible paths. We will show how to define the set of admissible paths as described in the paper.

\subsubsection{The minimal distance path}

The simplest example of a set of admissible paths is the set of minimal distance paths used by Chambers and Miller (2010). For a pair $x, y \in Z$, this is the set of all paths minimizing distance between $x$ and $y$ while remaining in $Z$. Suppose that $d(x, y)$ represents the Euclidean distance between $x$ and $y$. Formally, given a path $g$ we may define the length of path $g$ as follows. Let $k \in \mathbb{N}$, and consider any collection of points $t \in[0,1]^{k}$ for which for all $i, t_{i} \leq t_{i+1}$. Define $l_{t}(g)=\sum_{i=1}^{k-1} d\left(g\left(t_{i}\right), g\left(t_{i+1}\right)\right)$. Then define $l(g)=$ $\sup _{k \in \mathbb{N}} \sup _{\left\{t \in[0,1]^{k}: t_{i} \leq t_{i+1}\right\}} l_{t}(g)$. We now define $\mathcal{A}_{Z}(x, y) \equiv\left\{g \in \mathcal{P}_{Z}(x, y)\right.$ : $l(g) \leq l(h)$ for all $\left.h \in \mathcal{P}_{Z}(x, y)\right\}$. This set of admissible paths corresponds to the shortest paths in terms of Euclidean distance.

\subsubsection{The road based paths}

This next example is similar, except in that it relies on an existing infrastructure of roads. Formally, we may define $\mathcal{R}_{Z}(x, y) \subset \mathcal{P}_{Z}(x, y)$ to be a set of roads connecting households located at $x$ and $y$. (For this example to make sense, it must be the case that every residence lies on some road. ${ }^{17}$ ) We then define the set of admissible paths to be $\mathcal{A}_{Z}(x, y) \equiv\left\{g \in \mathcal{R}_{Z}(x, y): l(g) \leq\right.$ $l(h)$ for all $\left.h \in \mathcal{R}_{Z}(x, y)\right\}$. This example captures the possibility of measuring difficulty of travel by length of the shortest road between the two points, rather than the Euclidean distance between them.

\subsubsection{The shortest-time paths}

In this example, we suppose that $d: Z \times Z \rightarrow \mathbb{R}$ is a function specifying the travel time between any pair of points, as opposed to the geographic distance. It may be that one need not travel by road, and instead can travel by ferry,

\footnotetext{
${ }^{16}$ Continuity here is defined with respect to the Euclidean topology induced on $Z$.

${ }^{17}$ We also implicitly assume that all pairs of residences are connected by roads contained within the state. For cases in which this may be unrealistic, it is possible to include standard transportation paths, such as ferry and airplane routes.
} 
train, etc. We recommend that travel times be computed with respect to the same vehicle. These statistics are readily available; for example, Google Maps $^{18}$ specifies approximate travel times between any pair of residences in the United States. As in the distance minimizing case, we define $l_{t}(g)=$ $\sum_{i=1}^{k-1} d\left(g\left(t_{i}\right), g\left(t_{i+1}\right)\right)$. Then we define $l(g)=\sup _{k \in \mathbb{N}} \sup _{\left\{t \in[0,1]^{k}: t_{i} \leq t_{i+1}\right\}} l_{t}(g)$. We now define $\mathcal{A}_{Z}(x, y) \equiv\left\{g \in \mathcal{P}_{Z}(x, y): l(g) \leq l(h)\right.$ for all $\left.h \in \mathcal{P}_{Z}(x, y)\right\}$. The metric $d$ can represent some other relevant quantity besides travel times; indeed, it can be altogether arbitrary. ${ }^{19}$

\subsubsection{Pre-existing political subdivisions}

The laws of forty-four of the fifty states favor districting plans which keep intact pre-existing political subdivisions, such as counties, cities, and voting precincts. We can favor plans fulfilling this requirement by declaring the shortest paths within the political entity to be admissible. For a path $g \in \mathcal{P}_{Z}(x, y)$, define $l(g)$ as above (either using the minimal distance, travel time, or arbitrary metric). Let $\mathcal{S}$ be a set of subsets of $\mathcal{K}_{Z}$ representing the set of political subdivisions which should preferably be kept intact. For a given political subdivision $S \in \mathcal{S}$, let $\mathcal{P}_{S}(x, y) \equiv\left\{g \in \mathcal{P}_{Z}(x, y): g([0,1]) \in S\right\}$ be the set of paths contained within the political subdivision. Define $\mathcal{A}_{S}(x, y) \equiv$ $\left\{g \in \mathcal{P}_{S}(x, y): l(g) \leq l(h)\right.$ for all $\left.h \in \mathcal{P}_{S}(x, y)\right\}$ as the set of shortest paths in the political subdivision. Let $\mathcal{A}_{Z}^{*}(x, y) \subset \mathcal{P}_{Z}(x, y)$ be a set of admissible paths chosen by some method, such as in the prior examples. ${ }^{20}$ Define $\mathcal{A}_{Z}(x, y) \equiv \bigcup_{S \in \mathcal{S}} \mathcal{A}_{S}(x, y) \cup \mathcal{A}_{Z}^{*}(x, y)$. For example, a path might be considered admissible if either (a) it remains entirely within the city limits of Branson, Missouri, or (b) it is the shortest path as the crow flies.

\footnotetext{
${ }^{18}$ http://maps.google.com

${ }^{19} \mathrm{It}$ is important to point out that for a general metric, the length of the minimal path connecting $x$ and $y$ can be strictly greater than the metric-distance between $x$ and $y$. It is also possible that a state, while Euclidean compact, could nonetheless be non-compact under some metric. This is a technical issue which we do not address here. Our general definition does not preclude the set of admissible paths from being empty.

${ }^{20}$ While it would normally make sense for both $\mathcal{A}_{S}(x, y)$ and $\mathcal{A}_{Z}^{*}(x, y)$ to be defined with respect to the same metric, this need not be the case, as $\mathcal{A}_{Z}^{*}(x, y)$ may be defined in a general way.
} 


\subsubsection{Cores of prior districts}

The set of admissible paths can be defined to privilege districts which substantially preserve prior districts. Let a prior districting plan $\mathcal{D} \subset \mathcal{K}_{Z}$ be a set of districts such that $\bigcup_{D \in \mathcal{D}}=Z$ and, for all $D_{1}, D_{2} \in \mathcal{D}$ such that $D_{1} \neq D_{2}$, interior $\left(D_{1} \cap D_{2}\right)=\varnothing$. For each district $D \in \mathcal{D}$, define $\mathcal{P}_{D}(x, y) \equiv$ $\left\{g \in \mathcal{P}_{Z}(x, y): g([0,1]) \in D\right\}$ as the set of paths contained entirely within the district. Define $\mathcal{A}_{D}(x, y) \equiv\left\{g \in \mathcal{P}_{D}(x, y): l(g) \leq l(h)\right.$ for all $\left.h \in \mathcal{P}_{D}(x, y)\right\}$ as the set of minimal paths between two points $x, y \in D$. As above, let $\mathcal{A}_{Z}^{*}(x, y) \subset \mathcal{P}_{Z}(x, y)$ be a set of admissible paths chosen by some method. Define $\mathcal{A}_{Z}(x, y) \equiv \bigcup_{D \in \mathcal{D}} \mathcal{A}_{D}(x, y) \cup \mathcal{A}_{Z}^{*}(x, y)$. Under this definition, a path will be admissible if it was the shortest path between two people in the prior district or if it is the shortest path between them.

\subsection{The Measure}

As in Chambers and Miller (2010), we suppose that there is a density function $f \in \mathcal{F}$ representing a population distribution. For $f \in \mathcal{F}$, let $F$ be the associated probability measure, so that $F(K) \equiv \int_{K} f(x) d x$ represents the population of district $K$. A measure of compactness is a function $s_{Z}$ : $\mathcal{K}_{Z} \times \mathcal{F} \rightarrow \mathbb{R}_{+}$which associates a unique number to every pair of a district and population distribution.

We denote the set of paths connecting $x$ and $y$ and lying completely in $K$ as $\mathcal{P}_{Z}(x, y ; K)$. That is, $\mathcal{P}_{Z}(x, y ; K)=\left\{g \in \mathcal{P}_{Z}(x, y): g([0,1]) \subset K\right\}$. Given an exogenous set of admissible paths $\mathcal{A}_{Z}(x, y)$ for each $x$ and $y$, the compactness measure of $K$ is now easy to define. Let $\mathcal{A}_{Z \mid K} \equiv\{(x, y)$ : $\left.\mathcal{P}_{Z}(x, y ; K) \bigcap \mathcal{A}_{Z}(x, y) \neq \varnothing\right\}$. That is, $\mathcal{A}_{Z \mid K}$ is the set of pairs of points for which some admissible path between them lies in the parcel $K$. Let the indicator $1_{\mathcal{A}_{Z \mid K}}: K \times K \rightarrow \mathbb{R}$ be a function which returns a 1 if $(x, y) \in \mathcal{A}_{Z \mid K}$, and 0 otherwise. ${ }^{21}$ The generalized measure of compactness is given by:

$$
s_{Z}(K, f) \equiv \int_{K} \int_{K} \mathbf{1}_{\mathcal{A}_{Z \mid K}}(x, y) \frac{f(x) f(y)}{F(K)^{2}} d y d x
$$

The expression states that the measure of a district $K$ is essentially the

\footnotetext{
${ }^{21}$ In the continuous case described, it is unnecessary to consider the case where $x$ and $y$ are not distinct. In the discrete case, the indicator $\mathbf{1}_{\mathcal{A}_{Z \mid K}}$ must be defined so that it returns a 1 if $x=y$.
} 
probability that for a randomly chosen pair, ${ }^{22}$ an admissible path between that pair lies in the district.

\section{Discussion}

Measures based on minimal-distance paths (whether in terms of Euclidean distance, road-based paths, time-travel paths, or some other metric) have two features which we wish to note. First, these measures only consider paths which are entirely contained within the boundaries of the state. Paths which enter other states are irrelevant. Second, these measures penalize deviations sharply. If, for some pair of points, no acceptable path is completely contained within the district, the measures do not assess whether an acceptable path is "mostly" within the district.

\subsection{Out-of-state paths}

An example which illustrates this first feature is provided in Figure $5 .{ }^{23}$ Figure 5 (a) depicts a rectangular state that contains four equally-sized cities, two in the west, and two in the east. Thick lines represent the boundaries of the state; thin lines represent roads connecting the four cities. Perhaps because of a geographic limitation, such as a tall mountain range, there is only one road in the state which connects the cities in the north to the cities in the south. This road is located near the center of the state. The cities in the west are also connected by a relatively short road that passes through a neighboring state; the cities in the east are connected by a similar road.

Two possible districting plans are depicted in Figures 5(b) and 5(c). In Figure 5(b), the state is divided into northern and southern districts. Using the road-based path measure, each district receives a perfect score. To see why this is the case, note that, in both districts, the shortest road-based path connecting the two cities is an east-west road that is entirely contained within the district.

In Figure 5(c), the state is divided into western and eastern districts. Here the eastern district receives a less-than-perfect score of 0.5. To see why this is the case, consider a random pair of individuals living in the eastern district.

\footnotetext{
${ }^{22}$ Randomly chosen pair means independently drawn according to the probability measure induced by $f$.

${ }^{23}$ We thank an anonymous referee for providing this example.
} 


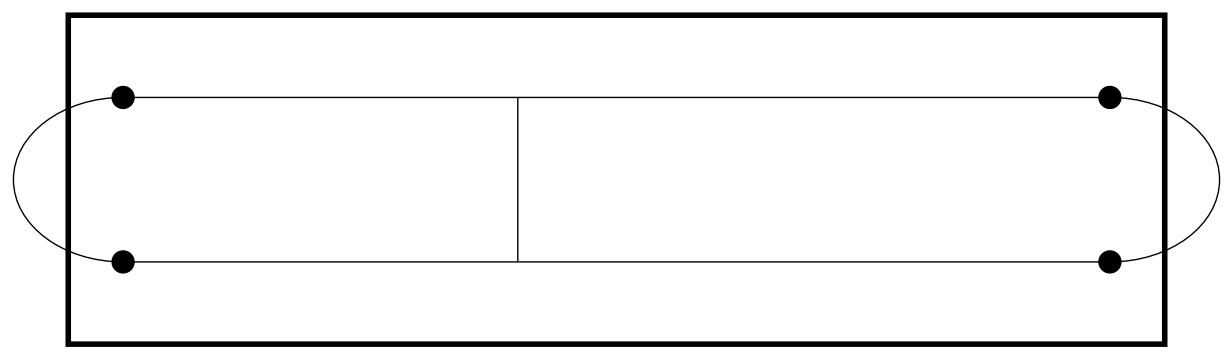

(a) State with four equally-sized cities

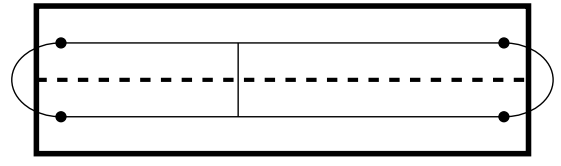

(b) Road-Based Measure: $(1,1)$

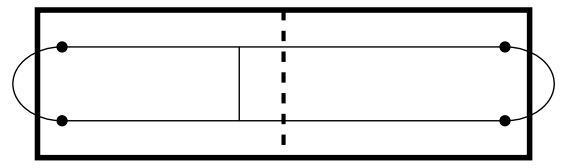

(c) Road-Based Measure: $(1,0.5)$

Figure 5: Road-based paths

With probability one-half, they live in the same city, and consequently the shortest road-based path connecting them will be in the district. However, it is equally likely that they live in different cities, in which case the shortest road-based path between them in the state enters the western district.

This example may be viewed as a criticism of road-based path measures. Arguably, the districting plan depicted in Figure 5(c) is preferable, because the eastern cities are relatively close, if one is willing to leave the state. However, this argument ignores concerns other than road distance. For example, when you leave the state, you need to drive past other people (or territory) to get to your neighbors in a different part of the district. This imposes a "cost" in terms of how neighborly you are with your fellow district members. While it is possible to modify the model so that this cost need not be infinite, the infinite cost is consistent with the legal requirement of "contiguity" found in most states, which requires it to be possible for an individual to travel between two points in a district without exiting the district.

Figure 6(a) depicts a state with eight equally-sized cities. Figures 6(b) and 6(c) show two possible districting plans; the former consists of an inner and an outer district while the latter consists of a northern and a southern district. If there is no cost to traveling out of the state, then one might argue that the districting plan depicted in Figure 6(b) is preferable because puts 


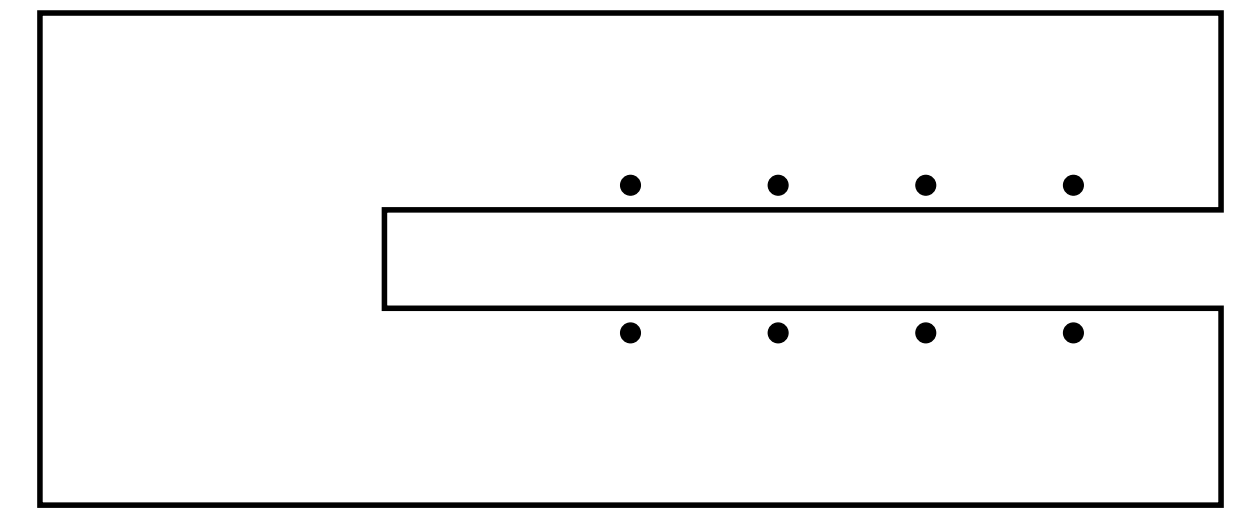

(a) State with eight equally-sized cities

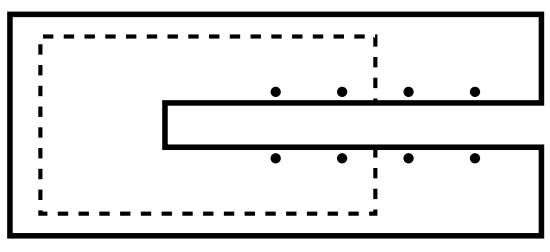

(b) Districting Plan 1

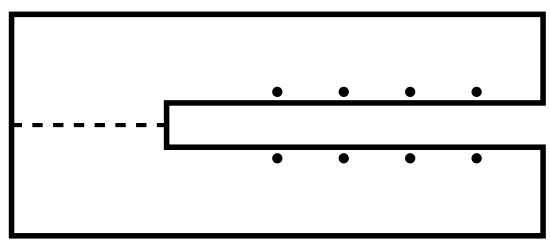

(c) Districting Plan 2

Figure 6: A state with two districts.

the eastern cities in one district and the western cities in another. However, the outer district is oddly-shaped because of the contiguity requirement. We believe that the districting plan depicted in Figure 6(c) is more natural.

\subsection{Penalizing deviations sharply.}

The second feature of minimal-distance path-based measures is that they penalize deviations sharply. To understand what we mean, let $\mathbf{1}_{\mathcal{A}_{Z \mid K}}(x, y)$ be the status of a pair of individuals. The measure considers only whether an admissible path between two individuals is entirely contained within the district. It does not give "partial credit" when a district "almost" contains an admissible path. That status of a pair of individuals is either one or zero. One might criticize the measure on the ground that it is not robust in the sense that a small change in the district may lead to a path not being included. While it is 
possible to amend the measure so that it gives "partial credit" (see Chambers and Miller, 2010), we believe that the stricter form of the measure we define is preferable in that it is both simpler to understand and to calculate.

Furthermore, we argue that robustness should be understood with respect to the entire measure, and not with respect to its' components. In the simplest case, when the population density function $f$ is non-atomic, a small change in the districting plan will only affect the status of a small proportion of pairs of individuals. Consequently, a small change in the districting plan will only lead to a small change in the measure of the districts. In practice, the population is finite and discrete; however, because the number of people in each district is large, and because relatively few people live at any single spot, a non-atomic density function is a fairly good approximation.

\section{Conclusion}

We have introduced the first family of compactness measures that can incorporate a wide range of internal geographic features. The measures in this family are the probability that a district contains an admissible path between a randomly selected pair of people. We have shown how the measure can account for roads, travel time, political boundaries, and prior districts. We have also shown that family of measures contains the path-based measure of Chambers and Miller (2010). This family of measures constitutes a systematic approach for assessing compliance with these districting principles.

The family of measures proposed in this paper is large. Furthermore, the construction of the measure allows us to combine sets of admissible paths; for example, we may define a path as admissible if it is either (a) contained within a city, or (b) contained within a prior district. Because we allow for an arbitrary set of admissible paths, every possible districting plan can be justified by some measure in this class. Consequently, it is important to be discriminating in one's choice of a measure. We envision two basic scenarios when the measure may be used.

First, the measure may be written into a state constitution or statute as a means to constrain the legislature's choice of districting plans. Similarly, the measure may be chosen by a state redistricting commission to distinguish between competing plans. In this case, it is important that the set of admissible paths be chosen in advance through a clear set of rules, and that these rules not change with each new districting plan. 
Second, the measure may be used by a judge or a social scientist to evaluate a districting plan approved by a particular state. In this case, the measure can serve as a check. For example, if the legislature were to justify a districting plan on the grounds that it preserved the cores of prior districts, the measure could be used to examine the validity of the claim. It may be possible to show that a district can not be justified by the goal of preserving the cores of prior districts. However, the measure can be used only to rule out explanations, and not to rule them in.

\section{References}

Altman, M. (1998): "Districting Principles and Democratic Representation," Ph.D. thesis, California Institute of Technology.

Boyce, R. R., and W. A. V. Clark (1964): "The Concept of Shape in Geography," Geographical Review, 54, 561-572.

Chambers, C. P., And A. D. Miller (2010): "A Measure of Bizarreness," Quarterly Journal of Political Science, 5, 27-44.

Cox, E. P. (1927): "A Method of Assigning Numerical and Percentage Values to the Degree of Roundness of Sand Grains," Journal of Paleontology, 1, $179-183$.

Fryer, JR., R. G., And R. Holden (2011): "Measuring the Compactness of Political Districting Plans," Journal of Law and Economics, 54(3), 493-535.

HARris, C. C. (1964): "A Scientific Method of Districting," Behavioral Science, 9, 219-225.

Hofeller, T., and B. Grofman (1990): "Comparing the Compactness of California Congressional Districts under Three Different Plans: 1980, 1982, and 1984."," in Political Gerrymandering and the Courts, ed. by B. Grofman. New York: Agathon.

KAISER, H. F. (1966): "An Objective Method for Establishing Legislative Districts," Midwest Journal of Political Science, 10, 200-213.

NCSL (2009): Redistricting Law 2010. National Conference of State Legislatures. 
Niemi, R. G., B. Grofman, C. Carlucci, and T. Hofeller (1990): "Measuring Compactness and the Role of a Compactness Standard in a Test for Partisan and Racial Gerrymandering," The Journal of Politics, 52, $1155-1181$.

Papayanopoulos, L. (1973): "Quantitative Principles Underlying Apportionment Methods," Annals of the New York Academy of Sciences, 219, $181-191$.

Polsby, D. D., and R. D. Popper (1991): "The Third Criterion: Compactness as a Procedural Safeguard Against Partisan Gerrymandering," Yale Law and Policy Review, 9, 301-353.

Reock, E. C. (1961): "A Note: Measuring Compactness as a Requirement of Legislative Apportionment," Midwest Journal of Political Science, 5, 70-74.

SchwartzberG, J. E. (1966): "Reapportionment, Gerrymanders, and the Notion of "Compactness"," Minnesota Law Review, 50, 443-452.

TAYLOR, P. J. (1973): "A New Shape Measure for Evaluation Electoral District Patterns," The American Political Science Review, 67, 947-950.

Weaver, J. B., and S. W. Hess (1963): "A Procedure for Nonpartisan Districting: Development of Computer Techniques," The Yale Law Journal, $73,288-308$.

Young, H. P. (1988): "Measuring the Compactness of Legislative Districts," Legislative Studies Quarterly, 13, 105-115. 